\title{
VIRTUAL COMPTON SCATTERING: RESULTS FROM JEFFERSON LAB
}

\author{
L. VAN HOOREBEKE \\ (FOR THE JEFFERSON LAB HALL A/VCS COLLABORATION) \\ Dept. Subatomic and Radiation Physics - RUG, Proeftuinstraat 86, 9000 Gent, \\ Belgium
}

\begin{abstract}
Virtual Compton Scattering off the proton has been studied at $Q^{2}$-values of 1.0 and $1.9(\mathrm{GeV} / \mathrm{c})^{2}$ in Hall $\mathrm{A}$ at the Thomas Jefferson National Accelerator Facility (JLab). Data were taken below and above the pion production threshold as well as in the resonance region. Results obtained below pion threshold at $Q^{2}=1.0(\mathrm{GeV} / \mathrm{c})^{2}$ are presented in this paper.
\end{abstract}

\section{Virtual Compton Scattering and Generalized Polarizabilities}

Virtual Compton Scattering (VCS) off the proton refers to the reaction $\gamma^{*} p \rightarrow \gamma p^{\prime}$ where $\gamma^{*}$ stands for an incoming virtual photon. Below pion threshold this reaction allows access to 6 new observables called Generalized Polarizabilities $(\mathrm{GPs})^{1,2}$. They are a function of $Q^{2}$ or $q$ (the modulus of the $\mathrm{cm}$ virtual photon three-momentum) only and are extensions of the electromagnetic polarizabilities $\bar{\alpha}$ and $\bar{\beta}$ obtained from Real Compton Scattering (RCS, $\left.Q^{2}=0(\mathrm{GeV} / \mathrm{c})^{2}\right)$. VCS off the proton can be accessed experimentally through the photon electro-production reaction $e p \rightarrow e^{\prime} p^{\prime} \gamma$. The real photon $\gamma$ (with $q^{\prime}$ the modulus of its $\mathrm{cm}$ three-momentum) can be emitted either by the incoming or the outgoing electron (the Bethe-Heitler contribution) or by the proton itself (the actual VCS process, containing the Born and Non-Born contributions). The Non-Born contribution contains the new physics and can be parametrized in terms of the 6 GPs. The Bethe-Heitler and Born $(B H+B)$ contributions can be accurately calculated using QED if the electromagnetic form factors of the proton are known. The $e p \rightarrow e^{\prime} p^{\prime} \gamma$ reaction cross section $d \sigma^{5} / d k_{l a b}^{\prime} d \Omega_{e^{\prime}}^{l a b} d \Omega_{p^{\prime}}^{c m}$ depending on $q, q^{\prime}, \epsilon$ (the virtual photon polarization), $\theta$ and $\varphi$ (the spherical angles indicating the $\mathrm{cm}$ direction of the outgoing photon relative to the virtual photon direction) can then conveniently be arranged as

$$
d^{5} \sigma_{e x p}=d^{5} \sigma_{B H+B}+\Phi q^{\prime} \Psi_{0}+\mathcal{O}\left(q^{2}\right)
$$

wherein $d^{5} \sigma_{B H+B}$ is the $B H+B$ cross section and $\Phi q^{\prime} \Psi_{0}$ is the lowest order polarizability effect where $\Phi$ is a phase space factor. $\Psi_{0}$ contains the GPs and 
in case of an unpolarized experiment at fixed $q$ one can write

$$
\frac{\Psi_{o}}{v_{2}}=\left[P_{L L}(q)-\frac{1}{\epsilon} P_{T T}(q)\right] \frac{v_{1}}{v_{2}}+P_{L T}(q)
$$

Here the $P_{I J}$ are structure functions which consist of linear combinations of five (out of six independent) GPs ${ }^{3}$ and $v_{1}$ and $v_{2}$ are known kinematical coefficients depending on $q, \epsilon, \theta, \varphi$. An experiment performed at fixed $q$ and $\epsilon$ allows to deduce $P_{L L}-\frac{1}{\epsilon} P_{T T}$ and $P_{L T}$.

First such results were obtained from data measured below pion production threshold at MAMI/Mainz ${ }^{4}$ at $q=600 \mathrm{MeV} / \mathrm{c}\left(Q^{2}=0.33(\mathrm{GeV} / \mathrm{c})^{2}\right)$. The VCS experiment (E93-050) ${ }^{6}$ performed at JLab has taken data below and above pion threshold at $Q^{2}=1.0$ and $1.9(\mathrm{GeV} / \mathrm{c})^{2}{ }^{8,9}$. Meanwhile, Pasquini et $a l .{ }^{5}$ have developed a dispersion relation formalism for VCS which can be used as a tool to extract GPs from data measured in the $\Delta(1232)$-resonance region and below. In this paper we restrict ourselves to JLab results obtained at $Q^{2}=1.0(\mathrm{GeV} / \mathrm{c})^{2}$ below pion threshold at the present stage of the analysis.

\section{Experimental Method and Data Analysis}

The experiment was performed in Hall A of JLab using a beam energy of $4 \mathrm{GeV}$ and a liquid hydrogen target. The scattered electron and recoil proton were detected in coincidence in two high-resolution magnetic spectrometers. Real photon production events were identified by missing-mass reconstruction. For the determination of the absolute cross section, the detector acceptance was accurately determined using an extensive Monte Carlo simulation ${ }^{7}$ which generates events starting from the $B H+B$ cross section and incorporates all resolution deteriorating effects. The cross section behaviour in the simulation was iterated using a lowest-order polarizability effect on top of the $B H+B$ cross section until convergence was obtained. Radiative corrections have been fully applied ${ }^{10}$.

\section{Results - Discussion}

The JLab VCS experiment allows to obtain cross sections in and out of the leptonic plane. The left-hand panel of fig. 1 shows the cross section determined in the leptonic plane as a function of $\theta$ for $q^{\prime}=45,75$ and $105 \mathrm{MeV} / \mathrm{c}$ (solid points), the full curve representing the $B H+B$ cross section. At low $q^{\prime}$ the data are in near agreement with the $B H+B$ cross section (as they should according to Eq. (1), this indicates the quality of the data analysis method),

baryons2002: submitted to World Scientific on May 3, 2002 

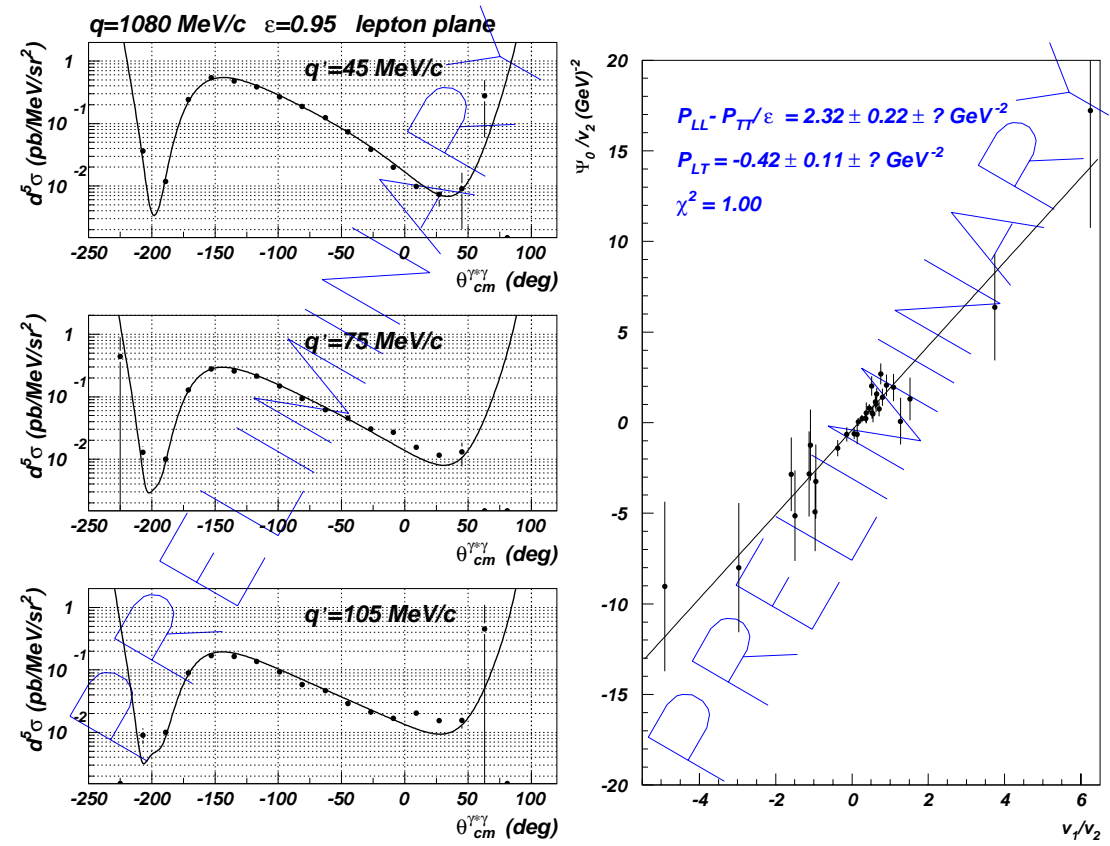

Figure 1. Left-hand panel: absolute cross sections (solid points) for the $e p \rightarrow e^{\prime} p^{\prime} \gamma$ reaction obtained in the leptonic plane as a function of $\theta$ at $q=1080 \mathrm{MeV} / \mathrm{c}\left(Q^{2}=0.923(\mathrm{GeV} / \mathrm{c})^{2}\right)$ and $\epsilon=0.95$, for three different values of $q^{\prime}$. The curve represents the $B H+B$ cross section. Right-hand panel: fit according to Eq. (2) to the data obtained in and out of the leptonic plane. The slope and intercept yield the two combinations of GPs. The errors indicated are statistical only, the systematic error (indicated by question marks) has still to be determined.

while with increasing $q^{\prime}$ one observes an increasing deviation from the $B H+B$ cross section due to the polarizability effect. This behaviour is also observed for the data obtained out of plane.

For each combination of angles $(\theta, \varphi) \Psi_{0}$ should fullfill Eq. (2). In case one makes the assumption that higher order terms do not play a role in Eq. (1), one can determine the value of $\Psi_{0}$ as the mean over the three $q^{\prime}$ values of $\left(d^{5} \sigma_{e x p}-d^{5} \sigma_{B H+B}\right) /\left[\Phi q^{\prime}\right]$ (low energy expansion (LEX) analysis, exactly the same approach was taken for the MAMI experiment ${ }^{4}$ ). Doing this for 12 data points obtained in the leptonic plane and 20 data points out of the leptonic plane, one can plot the left-hand side of Eq. (2) as a function of $v_{1} / v_{2}$, as shown in the right-hand panel of fig. 1. The data points are reasonably 
well aligned and a linear fit to the data yields $P_{L L}-\frac{1}{\epsilon} P_{T T}$ as slope and $P_{L T}$ as intercept. The indicated errors on the obtained values are statistical only, the systematic errors still have to be determined. The obtained values for the two structure functions are about $1 / 10$ of the values measured at $Q^{2}=0.33(\mathrm{GeV} / \mathrm{c})^{2}{ }^{4}$.

Concluding, it is clear that the "close to final" results presented here are very promising and indicate a strong $Q^{2}$ dependence of the structure functions $P_{L L}-\frac{1}{\epsilon} P_{T T}$ and $P_{L T}$. To finalize the analysis, the systematic errors have to be determined and additional stability checks of the data have to be performed. In addition to the LEX analysis, it is also planned to use the data obtained below pion threshold to extract GPs using the new dispersion relation formalism ${ }^{5}$.

\section{Acknowledgments}

This work was supported by DOE, NSF, by contract DE-AC05-84ER40150 under which the Southeastern Universities Research Association (SURA) operates the Thomas Jefferson National Accelerator Facility for DOE, by the French CEA, the Universite Blaise Pascal de Clermont-Ferrand and the CNRS/IN2P3 (France), the FWO-Flanders (Belgium), the BOF-Gent University (Belgium) and by the European Commission ERB FMRX-CT96-0008.

\section{References}

1. P.A.M. Guichon, G.Q. Liu and A.W. Thomas, Nucl. Phys. A 591, 606 (1995).

2. D. Drechsel, G. Knöchlein, A. Metz and S. Scherer, Phys. Rev. C 55, 424 (1997); D. Drechsel et al., Phys. Rev. C 57, 941 (1998).

3. P.A.M. Guichon and M. Vanderhaeghen, Prog. Part. Nucl. Phys. 41, 125 (1998).

4. J. Roche et al., Phys. Rev. Lett. 85, 708 (2000).

5. B. Pasquini et al., Eur. Phys. J. A11, 185 (2001), and also B. Pasquini, these proceedings.

6. P.Y. Bertin, P.A.M. Guichon and C. Hyde-Wright, spokespersons JLab E93-050 proposal, (1993).

7. L. Van Hoorebeke et al., in preparation.

8. H. Fonvieille, these proceedings.

9. L. Todor, these proceedings.

10. M. Vanderhaeghen et al., Phys. Rev. D 62, 025501 (2000).

baryons2002: submitted to World Scientific on May 3, 2002 